\title{
Fuzzy Optimal Control for Double Inverted Pendulum
}

\author{
Basil M. Al-Hadithi
}

José Manuel Andújar

\author{
Antonio Javier Barragán
}

Agustín Jiménez

\begin{abstract}
In this paper a fuzzy optimal control for stabilizing an upright position a double inverted pendulum (DIP) is developed and compared. Modeling is based on Euler-Lagrange equations. This results in a complicated nonlinear fast reaction, unstable multivariable system. Firstly, the mathematical models of double pendulum system are presented. The weight variable fuzzy input is gained by combining the fuzzy control theory with the optimal control theory. Simulation results show that the controller, which the upper pendulum is considered as main control variable, has high accuracy, quick convergence speed and higher precision.
\end{abstract}

Keywords-component; Takagi-Sugeno model, Linear quadratic regulator, double inverted pendulum

\section{INTRODUCTION}

As a nonlinear plant, DIP poses a challenging control problem. It seems to have been one of attractive tools for testing linear and nonlinear control laws [2] and [1]. Nearly all works on pendulum control concentrate on two problems: pendulums swing up control design and stabilization of the inverted pendulums. In [5] the stabilization control of DIP is developed. The authors linearize the DIP in one operating point which reduces its accuracy.

The classical control theory is based on design of controllers for stable systems which are in many cases more or less nonlinear. But it is difficult to use linearization methods in systems with high degree of nonlinearities. Therefore the need of nonlinear controllers arises.

To overcome limitations of the classical control theory, other methods are also introduced into the control design process, for example NN and fuzzy logic. Above all, fuzzy logic is very effective and its power has been demonstrated in various fields of system theory and applications where robustness is a very welcome property which is decisive for the choice of the controller.

Furuta et al. [4] designed a controller for DIP by means of the state-space approach and the minimal-order observer. Furuta et al. have designed and developed a digital controller for a DIP on inclined rail. The study based on fuzzy control theory has been done by a controller for stabilizing a doubleinverted pendulum at a upright position.

In this paper, fuzzy and optimal nonlinear controller is developed to stabilize a DIP minimizing an accumulative cost functional quadratic in states and controls. For linear systems, this leads to linear feedback control, which is found by solving a Riccati equation, and thus referred to as linear quadratic regulator (LQR). DIP, however, is a highly nonlinear system, and its linearization is far from adequate for control design purposes. Therefore, to solve the nonlinear optimal control problem, we will employ a LQR fuzzy controller. LQR is used to obtain the optimal solution at each fuzzy rule. Fuzzy control will deal with the nonlinearity of the DIP by adjusting the LQR parameters in each rule.

\section{MODELLING OF THE DOUBLE INVERTED PENDULUM}

The DIP system is shown in Fig. 1. A DIP of two links 11, 12 moves under the action of a single control input. Generalized coordinates $\theta_{1}(t)$ and $\theta_{2}(t)$ are attached to the system. The inputs are $\tau 1$ and $\tau 2$. The system with link li of mass mi $(\mathrm{kg})$ and length $\mathrm{li}(\mathrm{m}) ; \mathrm{i}=1 ; 2$; the unit of time is the second(s). Acceleration due to gravity is written as g. The mathematical model is derived from the Lagrange - Euler equation which describes mutual relations among kinetic, potential and external energy. All three components of energy must be balanced.

$$
M(\theta) \ddot{\theta}+C(\theta, \dot{\theta}) \dot{\theta}+B \dot{\theta}+g(\theta)=\tau
$$

$$
\begin{aligned}
& {\left[\begin{array}{cc}
M_{11} & M_{12} \\
M_{21} & M_{22}
\end{array}\right]\left[\begin{array}{l}
\ddot{\theta}_{1} \\
\ddot{\theta}_{2}
\end{array}\right]+} \\
& {\left[\begin{array}{cc}
\beta_{1}-h \dot{\theta}_{1} & +h \dot{\theta}_{2} \\
-\beta_{2}-h \dot{\theta}_{1} & \beta_{2}
\end{array}\right]\left[\begin{array}{l}
\dot{\theta}_{1} \\
\dot{\theta}_{2}
\end{array}\right]+\left[\begin{array}{l}
-g_{1} \\
-g_{2}
\end{array}\right]=\left[\begin{array}{l}
\tau_{1} \\
\tau_{2}
\end{array}\right]}
\end{aligned}
$$




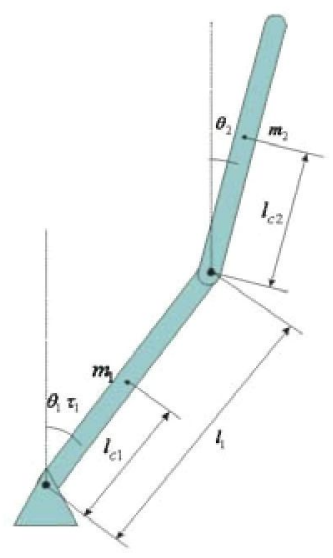

Figure 1. Double Inverted Pendulum

Where

$\mathrm{M}(\theta)$ is a $2 \times 2$ DIP inertia matrix

$C(\theta, \dot{\theta})$ is a $2 \times 2$ DIP centripetal and coriolis torques matrix $\beta_{1}$ and $\beta_{1}$ are the coefficients of viscous friction of the joints $\mathrm{g}(\theta)$ is a 2 -vector of gravitational torques

$$
\begin{aligned}
& M_{11}=m_{1} l_{c 1}^{2}+I_{1}+m_{2}\left(l_{1}^{2}+l_{1} l_{c 2} \cos \left(\theta_{1}-\theta_{2}\right)\right) \\
& M_{12}=m_{2} l_{1} l_{c 2} \cos \left(\theta_{1}-\theta_{2}\right)+m_{2} l_{c 2}^{2}+I_{2} \\
& M_{21}=m_{2} l_{1} l_{c 2} \cos \left(\theta_{1}-\theta_{2}\right) \\
& M_{22}=m_{2} l_{c 2}^{2}+I_{2} \\
& h=m_{2} l_{1} l_{c 2} \sin \left(\theta_{1}-\theta_{2}\right) \\
& g_{1}=m_{1} l_{c 1} g \sin \theta_{1}+m_{2} g\left(l_{c 2} \sin \theta_{2}+l_{1} \sin \theta_{1}\right) \\
& g_{2}=m_{2} l_{c 2} g \sin \theta_{2}
\end{aligned}
$$

For the equilibrium point

$\theta_{1}=\dot{\theta}_{1}=\ddot{\theta}_{1}=\theta_{2}=\dot{\theta}_{2}=\ddot{\theta}_{2}=0$

It can be deduced

$$
\tau_{10}=g_{10}=0, \tau_{20}=g_{20}=0
$$

The DIP under study has the following numerical values:

$$
\begin{array}{lllll}
m_{1}=4 & l_{1}=1 & l_{c 1}=0.5 & I_{1}=1 & \beta_{1}=0.75 \\
m_{2}=3 & l_{2}=1 & l_{c 2}=0.75 & I_{2}=0.5 & \beta_{2}=0.75
\end{array}
$$

We suppose that the action on the second axis is negligible $\tau_{2}=0$ and the stabilization of the pendulum at an upright position is made using only one input $\tau_{1}$.

\section{IDENTIFICATION OF T-S MODEL}

An interesting method of identification is presented in [13]. The idea is based on estimating the nonlinear system parameters minimizing a quadratic performance index. The method is based on the identification of functions of the following form:

$$
\begin{aligned}
& \mathrm{f}: \mathfrak{R}^{\mathrm{n}} \rightarrow \mathfrak{R} \\
& \mathrm{y}=\mathrm{f}\left(\mathrm{x}_{1}, \mathrm{x}_{2}, \ldots, \mathrm{x}_{\mathrm{n}}\right)
\end{aligned}
$$

Each IF-THEN rule $\mathrm{R}^{\mathrm{i} 1 \ldots \text { in }}$, for an $\mathrm{n}^{\text {th }}$ order system can be rewritten as follows:

$$
\begin{gathered}
S^{\left(i_{1} \ldots i_{n}\right)}: \text { if } x_{1} \text { is } M_{1}^{i_{1}} \text { and } x_{2} \text { is } M_{2}^{i_{2}} \text { and } \ldots x_{n} \text { is } M_{n}^{i_{n}} \\
\text { then } \hat{y}=p_{1}^{\left(i_{1} \ldots i_{n}\right)} x_{1}+p_{2}^{\left(i_{1} \ldots i_{n}\right)} x_{2}+\ldots+p_{n}^{\left(i_{1} \ldots i_{n}\right)} x_{n}
\end{gathered}
$$

Where the fuzzy estimation of the output is:

$$
\hat{y}=\frac{\sum_{i_{1}=1}^{r_{1}} \ldots \sum_{i_{n}=1}^{r_{n}} w^{\left(i_{1} \ldots i_{n}\right)}(x)\left[p_{1}^{\left(i_{1} \ldots i_{n}\right)} x_{1}+\ldots+p_{n}^{\left(i_{1} \ldots i_{n}\right)} x_{n}\right]}{\sum_{i_{1}=1}^{r_{1}} \ldots \sum_{i_{n}=1}^{r_{n}} w^{\left(i_{1} \ldots i_{n}\right)}(x)}
$$

Let $\mathrm{m}$ be a set of input/output system samples $\left\{x_{1 k}, x_{2 k}, \ldots, x_{n k}, y_{k}\right\}$. The parameters of the fuzzy system can be calculated as a result of minimizing a quadratic performance index:

$J=\sum_{k=1}^{N}\left(y_{k}-\hat{y}_{k}\right)^{2}=\|Y-X P\|^{2}$

where

$$
\begin{aligned}
Y & =\left[\begin{array}{llll}
y_{1} & y_{2} & \cdots & y_{N}
\end{array}\right]^{t} \\
P & =\left[p_{1}^{(1 \ldots 1)} p_{2}^{(1 \ldots 1)} \ldots p_{n}^{(1 \ldots 1)} \ldots p_{1}^{\left(r_{1} \ldots r_{n}\right)} \ldots p_{n}^{\left(r_{1} \ldots r_{n}\right)}\right]^{t}
\end{aligned}
$$

$$
X=\left[\begin{array}{c}
\beta_{1}^{(1 . .1)} x_{11} \ldots \beta_{1}^{(1 . .1)} x_{n 1} \cdots \beta_{1}^{\left(r_{1} . . r_{n}\right)} x_{11} \ldots \beta_{1}^{\left(r_{1} . r_{n}\right)} x_{n 1} \\
\vdots \\
\beta_{N}^{(1 . .1)} x_{1 N} \ldots \beta_{N}^{(1 . .1)} x_{n N} \cdots \beta_{N}^{\left(r_{1} . r_{n}\right)} x_{1 N} \ldots \beta_{N}^{\left(r_{1} . r_{n}\right)} x_{n N}
\end{array}\right]
$$

And

$$
\beta_{k}^{\left(i_{1} \ldots i_{n}\right)}=\frac{w^{\left(i_{1} \ldots i_{n}\right)}\left(x_{k}\right)}{\sum_{i_{1}=1}^{r_{1}} \ldots \sum_{i_{n}=1}^{r_{n}} w^{\left(i_{1} \ldots i_{n}\right)}\left(x_{k}\right)}
$$

If $\mathrm{X}$ is a matrix of complete rank, the solution is obtained as follows: 
$J=\|Y-X P\|^{2}=(Y-X P)^{t}(Y-X P)$

$\nabla J=X^{t}(Y-X P)=X^{t} Y-X^{t} X P=0$

$P=\left(X^{t} X\right)^{-1} X^{t} Y$

In the case of dynamic Systems represented by state variables, the system model is the following:

$\mathrm{x}^{\prime}=\mathbf{f}(x, \mathrm{u})$

$\mathrm{x}: \mathfrak{R} \rightarrow \mathfrak{R}^{\mathrm{n}}, \mathrm{u}: \mathfrak{R} \rightarrow \mathfrak{R}^{\mathrm{m}}, \mathrm{f}: \mathfrak{R}^{\mathrm{n}+\mathrm{m}} \rightarrow \mathfrak{R}^{n}$

T-S fuzzy model can be expressed as follows:

$$
\begin{gathered}
S^{\left(i_{1} \ldots i_{n}\right)}: \text { if } x_{1} \text { is } M_{1}^{i_{1}} \text { and } \ldots x_{n} \text { is } M_{n}^{i_{n}} \\
\text { then } x^{\prime}=A^{\left(i_{1} \ldots i_{n}\right)} x+B^{\left(i_{1} \ldots i_{n}\right)} u
\end{gathered}
$$

This is equivalent to $\mathrm{n}$ models in differential equation form:

$$
\begin{gathered}
S_{j}^{\left(i_{1} \ldots i_{n}\right)}: \text { if } x_{1} \text { is } M_{1}^{i_{1}} \text { and } \ldots x_{n} \text { is } M_{n}^{i_{n}} \text { then } \\
x_{j}^{\prime}=a_{j 1}^{\left(i_{1} \ldots i_{n}\right)} x_{1}+a_{j 2}^{\left(i_{1} \ldots i_{n}\right)} x_{2}+\ldots+a_{j n}^{\left(i_{1} \ldots i_{n}\right)} x_{n} \\
+b_{j 1}^{\left(i_{1} \ldots i_{n}\right)} u_{1}+\ldots+b_{j m}^{\left(i_{1} \ldots i_{n}\right)} u_{m}
\end{gathered}
$$

Applying the previous method for each one of these models, we get:

$$
\begin{aligned}
Y_{j} & =\left[\begin{array}{llll}
x_{j 1}^{\prime} & x_{j 2}^{\prime} & \cdots & x_{j N}^{\prime}
\end{array}\right]^{t} \\
P_{j} & =\left[\begin{array}{lll}
a_{j 1}^{(1 \ldots 1)} \ldots a_{j n}^{(1 \ldots 1)} & (1 \ldots 1) & \ldots b_{j 1}^{(1 \ldots 1)} \\
& \ldots & a_{j 1}^{\left(\eta_{1} \ldots r_{n}\right)} \ldots b_{j m}^{\left(n_{1} \ldots r_{n}\right)}
\end{array}\right]^{t}
\end{aligned}
$$

and each row of the $\mathrm{X}$ matrix will be

$$
\begin{aligned}
X_{k} & =\left[\beta_{k}^{(1 . .1)} x_{1 k} \ldots \beta_{k}^{(1 . .1)} x_{n k} \beta_{k}^{(1.1)} u_{1 k} \ldots \beta_{k}^{(1 . .1)} u_{m k}\right. \\
& \left.\ldots \beta_{k}^{\left(\eta_{1} . r_{n}\right)} x_{1 k} \ldots \beta_{k}^{\left(\eta_{1} . r_{n}\right)} u_{m k}\right]
\end{aligned}
$$

As it can be seen, the $X$ matrix is the same for $\mathrm{j}$ models, therefore they can be grouped as follows:

$Y=\left[\begin{array}{lll}Y_{1} & \cdots & Y_{n}\end{array}\right]$

$P=\left[\begin{array}{lll}P_{1} & \cdots & P_{n}\end{array}\right]$

And the solution is still:

$$
P=\left(X^{t} X\right)^{-1} X^{t} Y
$$

\section{A. T-S model for the double inverted pendulum}

In order to obtain T-S model for the DIP, we have to take into consideration the following variables:

$$
x=\left[\begin{array}{llll}
\theta_{1} & \dot{\theta}_{1} & \theta_{2} & \dot{\theta}_{2}
\end{array}\right]^{t}
$$

$$
\begin{aligned}
\text { If } x_{1} \text { is } M_{1}^{i_{1}} \text { and } \ldots x_{4} \text { is } M_{4}^{i_{4}} \text { then } \\
x^{\prime}=\left[\begin{array}{cccc}
0 & 1 & 0 & 0 \\
a_{21}^{\left(i_{1} i_{2} i_{3} i_{4}\right)} & a_{22}^{\left(i_{1} i_{2} i_{3} i_{4}\right)} & a_{23}^{\left(i_{1} i_{2} i_{3} i_{4}\right)} & a_{24}^{\left(i_{1} i_{2} i_{3} i_{4}\right)} \\
0 & 0 & 0 & 1 \\
a_{41}^{\left(i_{1} i_{2} i_{3} i_{4}\right)} & a_{42}^{\left(i_{1} i_{2} i_{3} i_{4}\right)} & a_{43}^{\left(i_{1} i_{2} i_{3} i_{4}\right)} & a_{44}^{\left(i_{1} i_{2} i_{3} i_{4}\right)}
\end{array}\right] x \\
+\left[\begin{array}{c}
0 \\
b_{21}^{\left(i_{1} i_{2} i_{3} i_{4}\right)} \\
0 \\
b_{41}^{\left(i_{1} i_{2} i_{3} i_{4}\right)}
\end{array}\right] \tau_{1}
\end{aligned}
$$

Five second order polynomial fuzzy sets have been used. They are centred in $-\frac{\pi}{6},-\frac{\pi}{12}, 0, \frac{\pi}{12}, \frac{\pi}{6}$ for angles and three other ones centred in $-3,-1.5,0,1.53$ for their derivatives as shown in Fig. 2.

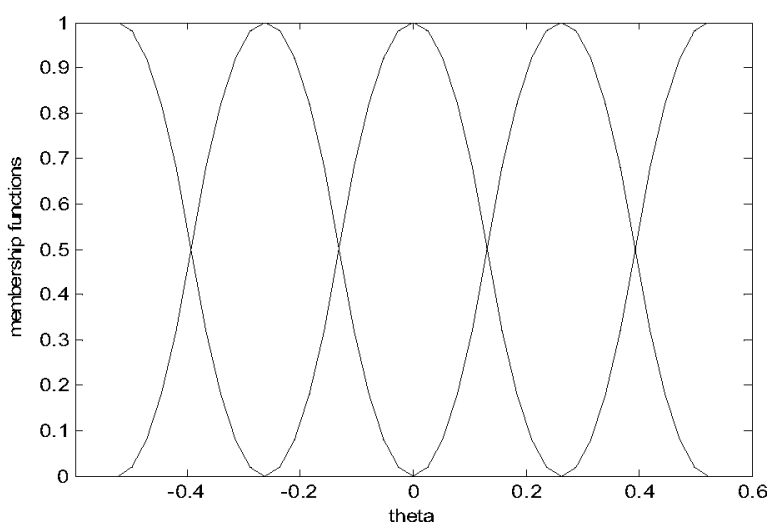

Figure 1. Membership functions

Applying the method we obtain 


$$
\begin{aligned}
& S^{(1111)}: \text { if } x_{1} \text { is } M_{1}^{1} \text { and } x_{2} \text { is } M_{2}^{1} \text { and } x_{3} \text { is } M_{3}^{1} \text { and } x_{4} \text { is } M_{4}^{1} \text { then } \\
& x^{\prime}=\left[\begin{array}{cccc}
0 & 1 & 0 & 0 \\
3.0724 & -0.8453 & 6.2471 & 0.5560 \\
0 & 0 & 0 & 1 \\
5.2168 & 1.2170 & -5.2476 & -0.9061
\end{array}\right] x+\left[\begin{array}{c}
0 \\
-0.3769 \\
0 \\
0.3903
\end{array}\right] \tau_{1} \\
& \vdots \\
& S^{(5555)}: \text { if } x_{1} \text { is } M_{1}^{5} \text { and } x_{2} \text { is } M_{2}^{5} \text { and } x_{3} \text { is } M_{3}^{5} \text { and } x_{4} \text { is } M_{4}^{5} \text { then } \\
& x^{\prime}=\left[\begin{array}{cccc}
0 & 1 & 0 & 0 \\
3.0771 & -0.8371 & 6.2498 & 0.5668 \\
0 & 0 & 0 & 1 \\
5.2122 & 1.2115 & -5.2485 & -0.9152
\end{array}\right] x+\left[\begin{array}{c}
0 \\
-0.3752 \\
0 \\
0.3874
\end{array}\right] \tau
\end{aligned}
$$

$$
\begin{gathered}
S^{(555)}: \text { if } x_{1} \text { is } M_{1}^{5} \text { and } x_{2} \text { is } M_{2}^{5} \text { and } x_{3} \text { is } M_{3}^{5} \text { and } x_{4} \text { is } M_{4}^{5} \text { then } \\
x^{\prime}=\left[\begin{array}{cccc}
0 & 1 & 0 & 0 \\
3.0771 & -0.8371 & 6.2498 & 0.5668 \\
0 & 0 & 0 & 1 \\
5.2122 & 1.2115 & -5.2485 & -0.9152
\end{array}\right] x+\left[\begin{array}{c}
0 \\
-0.3752 \\
0 \\
0.3874
\end{array}\right] \tau_{1}
\end{gathered}
$$

\section{DESIGN OF FUZZY OPTIMAL CONTROLLER}

Together with the proposed estimation method, the wellknown LQR method might be an appropriate choice [6]. If the system can be represented in state space form:

$x^{\prime}=A x+B u$

$x \in \mathfrak{R}^{n}, u \in \mathfrak{R}^{m}, A \in \mathfrak{R}^{n x n}, B \in \mathfrak{R}^{n x m}$

The objective is to find the control action $\mathbf{u}(\mathbf{t})$ to transfer the system from any initial state $x\left(t_{0}\right)$ to some final state $x(\infty)=0$ in an infinite time interval, minimizing a quadratic performance index of the form:

$J=\int_{t_{0}}^{\infty}\left(x^{t} Q x+u^{t} R u\right) d t$

where $Q \in \mathfrak{R}^{n x n}$ is a symmetric matrix, at least positive a semi-definite one $R \in \mathfrak{R}^{m x m}$ is also a symmetric positive definite matrix. The optimal control law is then computed as follows:

$u(t)=-K x(t)$

$K=R^{-1} B^{t} L$

where the matrix $L \in \mathfrak{R}^{n x n}$ is a solution of the Riccati equation:
$0=-Q+L B R^{-1} B^{t} L-L A-A^{t} L$

The LQR methodology can be applied for each subsystem using a common state weighting matrix $Q$ and input matrix $R$ for all the rules. Thus, Riccati equation is solved for each subsystem as follows:

$$
\begin{gathered}
0=-Q+L^{\left(i_{1} \ldots i_{n}\right)} B^{\left(i_{1} \ldots i_{n}\right)} R^{-1} B^{\left(i_{1} \ldots i_{n}\right) t} L^{\left(i_{1} \ldots i_{n}\right)}- \\
L^{\left(i_{1} \ldots i_{n}\right)} A^{\left(i_{1} \ldots i_{n}\right)}-A^{\left(i_{1} \ldots i_{n}\right) t} L^{\left(i_{1} \ldots i_{n}\right)}
\end{gathered}
$$

Then the state feedback gain vector can be obtained as follows:

$$
\begin{aligned}
& K^{\left(i_{1} \ldots i_{n}\right)}=\left[\begin{array}{llll}
k_{1}^{\left(i_{1} \ldots i_{n}\right)} & k_{2}^{\left(i_{1} \ldots i_{n}\right)} & \ldots & k_{n}^{\left(i_{1} \ldots i_{n}\right)}
\end{array}\right]= \\
& =R^{-1} B^{\left(i_{1} \ldots i_{n}\right) t} L^{\left(i_{1} \ldots i_{n}\right)} \\
& C^{\left(i_{1} \ldots i_{n}\right)} \text { :if } x_{1} \text { is } M_{1}^{i_{1}} \text { and } \ldots x_{n} \text { is } M_{n}^{i_{n}} \\
& \text { then } u=-K^{\left(i_{1} \ldots i_{n}\right)} x
\end{aligned}
$$

In the case of the DIP under study

$$
\begin{aligned}
& C^{(1111)} \text {.if } x_{1} \text { is } M_{1}^{1} \text { and } \cdots x_{4} \text { is } M_{4}^{1} \text { then } \\
& \tau_{1}=-\left[\begin{array}{llll}
79.9902 & 31.5107 & 42.4132 & 18.1586
\end{array}\right] x \\
& \vdots \\
& C^{(5555)} \text { :if } x_{1} \text { is } M_{1}^{5} \text { and } \cdots x_{4} \text { is } M_{4}^{3} \text { then } \\
& \tau_{1}=-\left[\begin{array}{llll}
80.1785 & 31.6095 & 42.4828 & 18.2289
\end{array}\right] x \\
& \text { V. SIMULATION RESULTS }
\end{aligned}
$$

The controller was tested under different initial conditions, varying intervals of universes of discourse. Fig. 3 and 4 show the evolution $\theta_{1}, \theta_{2}$ from conditions of $20^{\circ}$ and $-10^{\circ}$ respectively. 


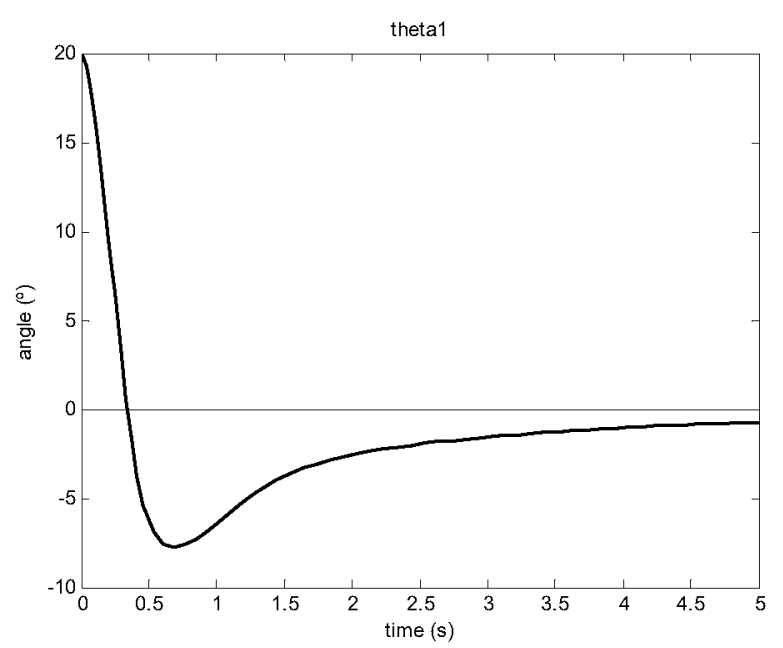

Figure 2. Evolution of $\theta_{1}$

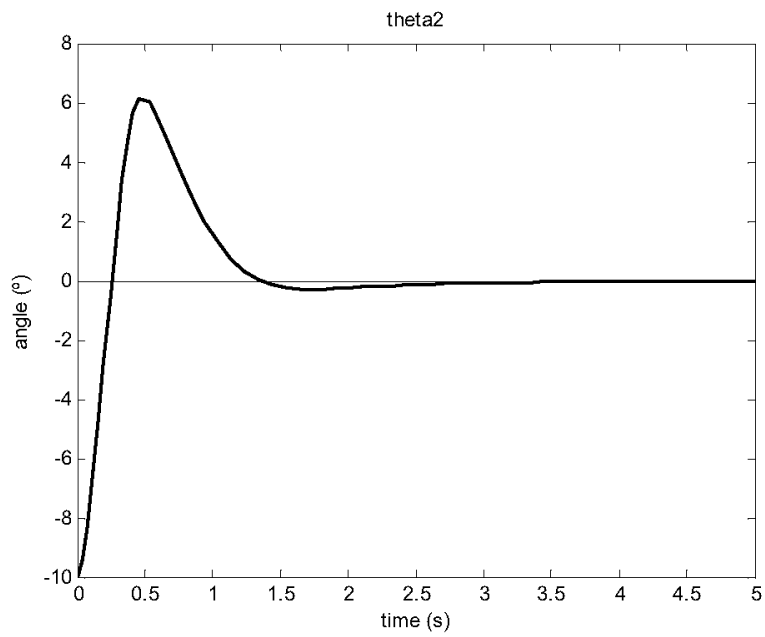

Figure 3. Evolution of $\theta_{2}$

\section{CONCLUSIONS}

In this paper an algorithm, using fuzzy optimal control for stabilizing at an upright position a double inverted pendulum (DIP), is developed. Modeling is based on Euler-Lagrange equations. This results in a complicated nonlinear fast reaction, unstable multivariable system. Simulation results show that the controller, which the upper pendulum is considered as main control variable, has high accuracy and quick convergence speed and higher precision. The control result can be expanded the control of multilevel inverted Pendulum, and have a guiding meaning in the control of other unstable system.

\section{REFERENCES}

[1] Brockett, R.W., Li, H.: A light weight rotary double pendulum: maximizing the domain of attraction. In Proceedings of the 42nd IEEE Conference on Decision and Control, Maui, Hawaii, December (2003).

[2] Furuta, K., Okutani, T., Sone, H.: Computer control of a double inverted pendulum. Computer and Electrical Engineering, 5, pp 67-84 (1978).

[3] Hornik, K., Stinchcombe, M., White, H.: Multilayer feedforward neural networks are universal approximators. Neural Networks, 2, pp 359-366 (1989).

[4] Furuta, K., Okutani, T., Sone, H.: Computer control of a double inverted pendulum, Comput. Electr. Eng. 5, pp 67-84.
[5] Li, Q.-R., Tao. W.-H., Na, S., Zhang, C.-Y., Yao, L.-H.: Stabilization Control of Double Inverted Pendulum System, The 3rd Intetnational Conference on Innovative Computing Information. and Control (ICICIC'08).

[6] Luo, C., Hu, D., Pang, Y., Zhu, X., Dong, G.: Fuzzy control of a quintuple inverted pendulum with the LQR method and 2-ary fuzzy piecewise interpolation function, Proceedings of the 5th IEEE Conference on Decision \& Control FrIP9.6 Manchester Grand Hyatt Hotel San Diego, CA, USA, December, pp 13-15 (2006).

[7] Horikawa, S.I., Furuhashi, T. Uchikawa, Y.: On fuzzy modeling using neural networks with the back propagation algorithm. IEEE Trans. Neural Networks, 3, pp 801-806 (1992).

[8] Lin, C.T., Lee C.S.G.: Neural network based fuzzy logic control and decision systems. IEEE Trans. Computers, 40, pp 1320-1336 (1992).

[9] Patricar, A., Provence, J.: A self-organizing controller for dynamic processes using neural networks. Intl. Joint Conf. Neural Networks, 3, pp 359364 (1990).

[10] Takagi, H., Hayashi, I.: NN-driven fuzzy reasoning. Intl. J. Approximate Reasoning, 5, pp 191-212 (1991).

[11] Kyung, K.H., Lee, B.H.: Fuzzy rule base derivation using neural network based fuzzy logic controller by self-learning. Proc. IECON'93, 1, pp 435-440 (1993).

[12] Sakai, S., Takahama, T.: Learning fuzzy control rules for inverted pendulum by simplex method. Proc. of the13th Fuzzy System Symposium, pp 61-64 (1997).

[13] Takagi, T., and Sugeno, M. Fuzzy identification of systems and its applications to modeling and control. IEEE Trans. Syst., Man, Cybern., vol. SMC-15, no. 1, pp 116-132, Jan. (1985) 